\title{
Early-warning system for safe drinking-water: A domain-specific modelling approach
}

\author{
Syed Imran
}

Cork Constraint Computation Centre, UCC

\section{Introduction}

The quality of drinking water in Ireland was brought forcefully to the attention of the Irish public with the outbreak of cryptosporidiosis in Galway City during 2007, which affected more than 90,000 people, causing illness in over 240 people, and led to the imposition of a boil water notice in Galway City for 5 months during the peak tourist season. In 2008, the Irish Environmental Protection Agency (EPA) identified 36\% of public water supplies (339 supplies) that required detailed profiling, that is, the representation of all the physical items that may constitute the drinking water treatment plant in order to ensure their capability to provide clean and wholesome drinking water. These water supplies, which were included on a Remedial Action List, required a range of actions to their drinking water treatment plants to ensure they could achieve this.

The aim is to develop a novel Early Warning System for water treatment plants. Its main goal is to connect people who are working in different organizations but act as one system for the provision of safe drinking water in the Republic of Ireland, i.e., State Agencies such as the Environmental Protection Agency, the Health Service Executive, Local Authorities, Water Treatment Plant Personnel, National and Private Laboratories. An additional goal is to assess the hazard level that is to indicate a seriousness of vulnerability problem that needs timely intervention for each water treatment plant, which are registered in the Early Warning System and to disseminate timely warnings to all stakeholders associated with a water treatment plant.

An important component of the Early Warning System is the one which will allow its users to systematically collect and represent their knowledge about risks and vulnerabilities of water treatment plants. The goal of my research is the realisation of this component by developing a graphical language able to represent concepts related to the domain of water treatment plants operations and safety. The graphical language will be an integrated component of an Early Warning System. The models specified by the graphical language will represent different facets of the domain and executable code will be generated automatically. In short, the objective of my research is to develop a Domain Specific Modeling 
language as a component. In order to allow the users to interact with this component of the Early Warning System, a dedicated software editor will be developed.

To achieve this, Domain Specific Modeling approach has been used to define a graphical language as a means to identify and represent the risks related to drinking water treatment and supply. The language aims to design and develop systems that will capture the complexity of drinking water systems. It will also facilitate the stakeholder's contribution in capturing the knowledge on potential risks to their drinking water supplies and allows earlier intervention to reduce the potential consequences of hazards on the treatment plant. The aim is to develop a language that can be used by both technical experts and non-technical personnel.

\section{What is Domain Modeling?}

Domain modeling can facilitate the translation of knowledge from the real life situations into visual graphical models. In order to develop a Domain Specific Modeling language, an extensive knowledge and understanding of the particular area or domain is required. The involvement of domain experts is essential to perform the detailed analysis. Several visits were made to local authority drinking water treatment plants that co-operated with this research who understood the benefits to their organisation from the outputs of this research. Detailed information on drinking water treatment plants was acquired and documented. An overall view for specifying the components of Domain Specific Modeling language is illustrated in Figure 1 on p.88. The physical entities and processes involved in the treatment of drinking water, and the hazards and vulnerabilities that can affect its safety and security were identified, based on system safety engineering approaches. The identified and validated concepts are then used to specify the components of our Domain Specific Modeling language.

\section{Dedicated Editor}

The objective is to provide a customized view of the drinking water treatment facility and an ability to carry out a risk analysis of it. The 'dedicated' software editor will serve as a platform for performing the risk analysis on different scenarios that may threaten the drinking water quality supplied from the water treatment plant. The editor will also provide the features to perform the profiling.

The 'dedicated' editor will consist of a set of icons that can be graphically dragged and dropped to generate the drinking water treatment plant profile applicable to the particular plant as it exists on the ground. It will also allow the validation of the drinking water treatment plant profile and the specific set of hazards that may threaten the plant. 


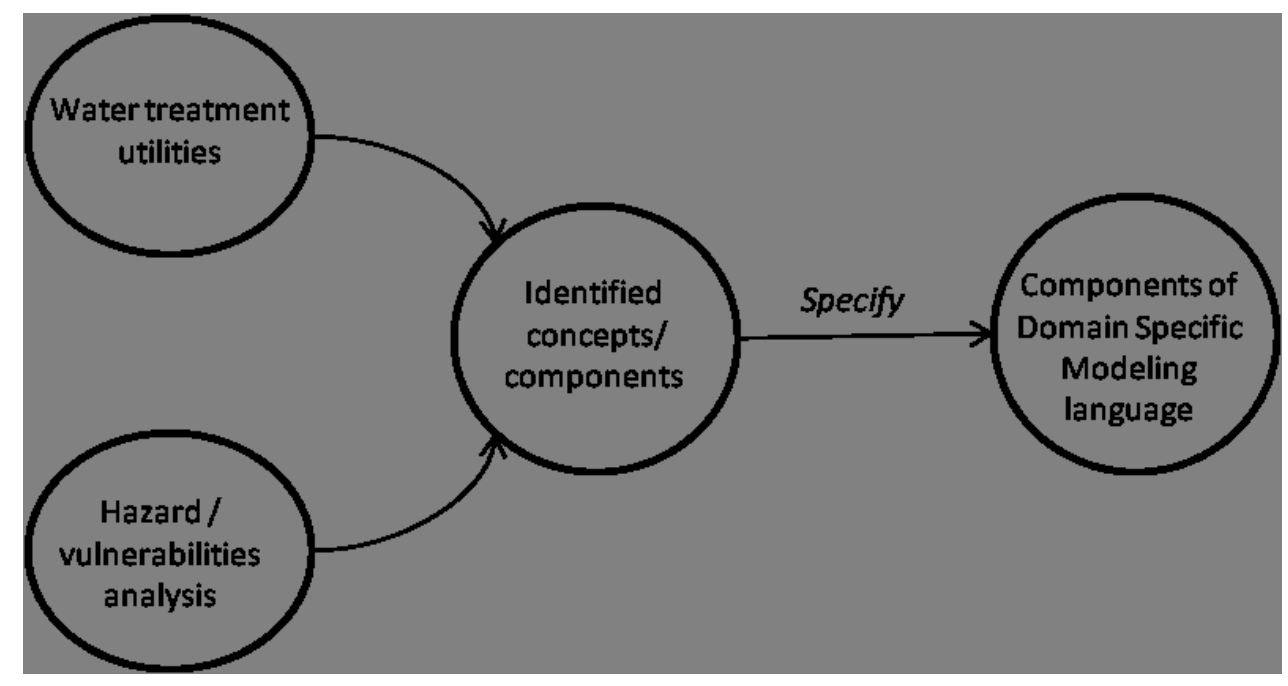

Figure 1: Software development process for specifying the artefacts of Domain Specific Modeling Language.

\section{Summary}

The goal of this research is to develop a knowledge modeling tool and its dedicated editor. The tool is based on Domain Specific Modeling approach and serves as a prominent component of a whole early warning system. Many technical and research challenges emerged during the development phase and require further research in effective performance and improved validation.

Software that serves as an Early Warning System in the area of drinking water treatment and supply will be a very useful contribution in supporting proactive risk management and its practical application by range of organisations in Ireland and would enable the provision of wholesome and clean drinking water to all consumers.

Syed Imarn is a student from the Cork Constraint Computation Centre of UCC under the supervision of Dr. loannis Dockas. This work is supported by the research project SCEWA (Grant No 2007-DRP-2-S5), funded by the Irish Environmental Protection Agency under the DERP grand scheme. 